\title{
HRTEM Characterization and Simulation of Ag Nanoplates
}

\author{
Natalia D. Aagaard ${ }^{1,2 *}$, Julio C. Azcárate ${ }^{1}$, Eugenia Zelaya ${ }^{1 .}$ \\ 1. Centro Atómico Bariloche, Bariloche, Rio Negro Argentina. CONICET. \\ 2. Facultad de Ciencias Exactas, Universidad Nacional de La Plata, La Plata, Argentina. \\ *Corresponding author: natalia.aagaard@cab.cnea.gov.ar.
}

Metallic nanoplates are extremely anisotropic particles. This characteristic makes then particularly interesting due to their plasmonic properties. The silver nanoplates also refereed as nanoprism or AgNPls presents very attractive properties like "hot spots". These properties are very useful in the design of plasmonic sensors or photocatalysis. The intensity and position of the absorption plasmonic band is highly dependent of the shape and size of the particles. In this work, the AgNPs were synthetized by reduction of silver salt $\left(\mathrm{AgNO}_{3}\right)$ in aqueous solution. Fig. 1a and 1b show the typical shape and size distribution of the obtained AgNPls. The thickness is rather constant; however, the lateral dimensions present a high dispersion. Moreover, the smaller nanoplates present an hexagonal shape instead a triangular one (Fig. 1c). The selected area diffraction patterns were describe in previous works like forbidden spots $1 / 3\{422\}$ of a face center cubic (fcc) supercell [1]. However, recent works describe these kind of AgNpls like mixed fcc and hexagonal closest packed structure (hcp). In other words, it is also possible to describe a lamellar fcc structure with stacking faults or twins in $\langle 110\rangle$ with basal planes $\{111\}$ over the bigger faces [2].

In this work, we simulate different kinds of fcc and hep stacking sequences and compare with actual high resolution transmission electron microscopy (HRTEM) images in order to better characterize the internal structure of the AgNpls. The samples were observed in a FEI Tecnai F20 G2 transmission electron microscope, with a spherical aberration constant of $0.5 \mathrm{~mm}$ and a point resolution of $0.14 \mathrm{~nm}$ operating at $200 \mathrm{kV}$. The simulations were performed using JEMS software and the parameters used in the FEI Tecnai F20 G2 microscope. The Fig. 2a shows the simulation of an fcc structure in [110] zone axis that is clearly not compatible with the HRTEM image showed in Fig. 1c. Moreover, the simulation of hcp structure in [0001] zone axis of Fig. 2b shows a very similar contrast to the images of the particles in Fig. 1c. In addition, it could be appreciate in Fig. 2 the black-white relative to the simulated atoms position can invert contrast when the defocus is changed. The simulations also showed that the contrast does not change no matter which high the hcp structure is stacked into the fcc structure. However, the introduction of an extra hcp plane or a twin could change the contrast dramatically. Moreover, the simulations shows that at least a twin or stacking fault is needed in order to obtain the contrast showed in the HRTEM images of Fig. 1c. Finally, it should be mention that the contrast is inverted with a presence of a twin (Fig. 2c and 2d). This result seems to be compatible with the presence of two particles with the opposite contrast in Fig 1c. All these analysis helps to conclude the importance of the simulation analysis of HRTEM images. Moreover, when the dimension of the particles makes particularly difficult to obtain a HRTEM image with the adequate resolution of the side view of the particles.

\section{References:}

[1] V Germain et. al, J. Phys. Chem. B 107 (2003), 8717-8720.

[2] D Aherne et.al, Adv. Funct. Mater. 18 (2008), 2005-2016. 

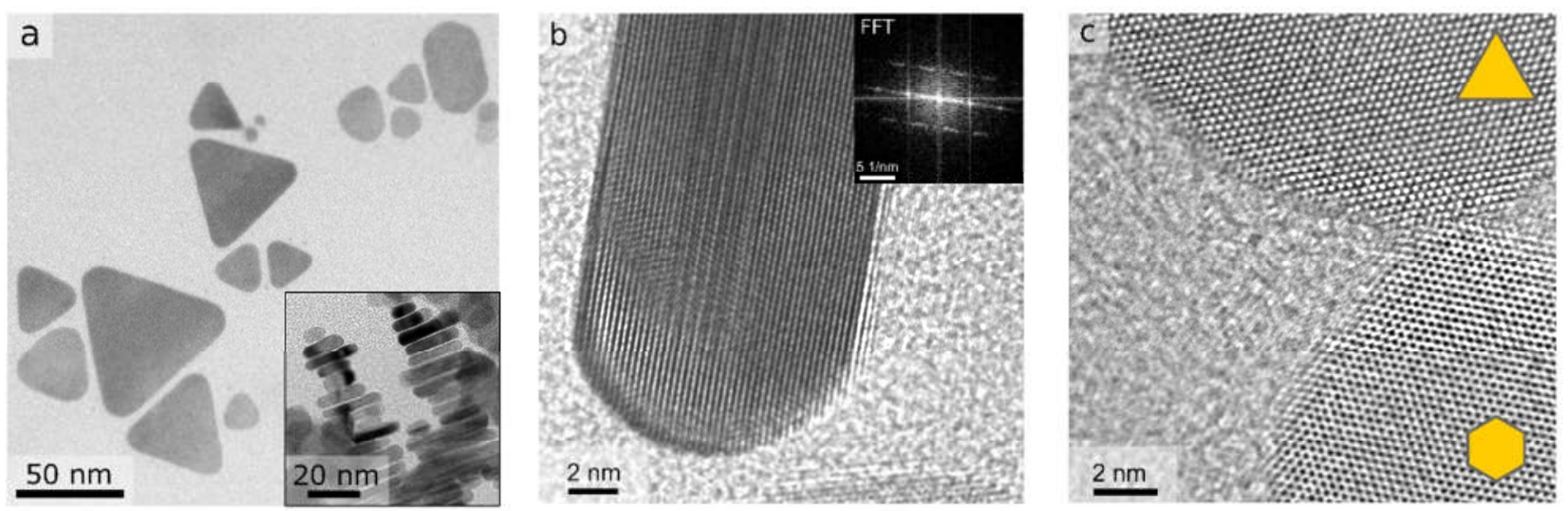

Figure 1. a. Bright field image of the nanoplates in plane view. The inset shows a side view of the AgNPls. b. HRTEM image of the side view of one AgNpls. c. HRTEM image of two particles (one with an hexagonal shape and the other with triangular shape) with opposite contrast.
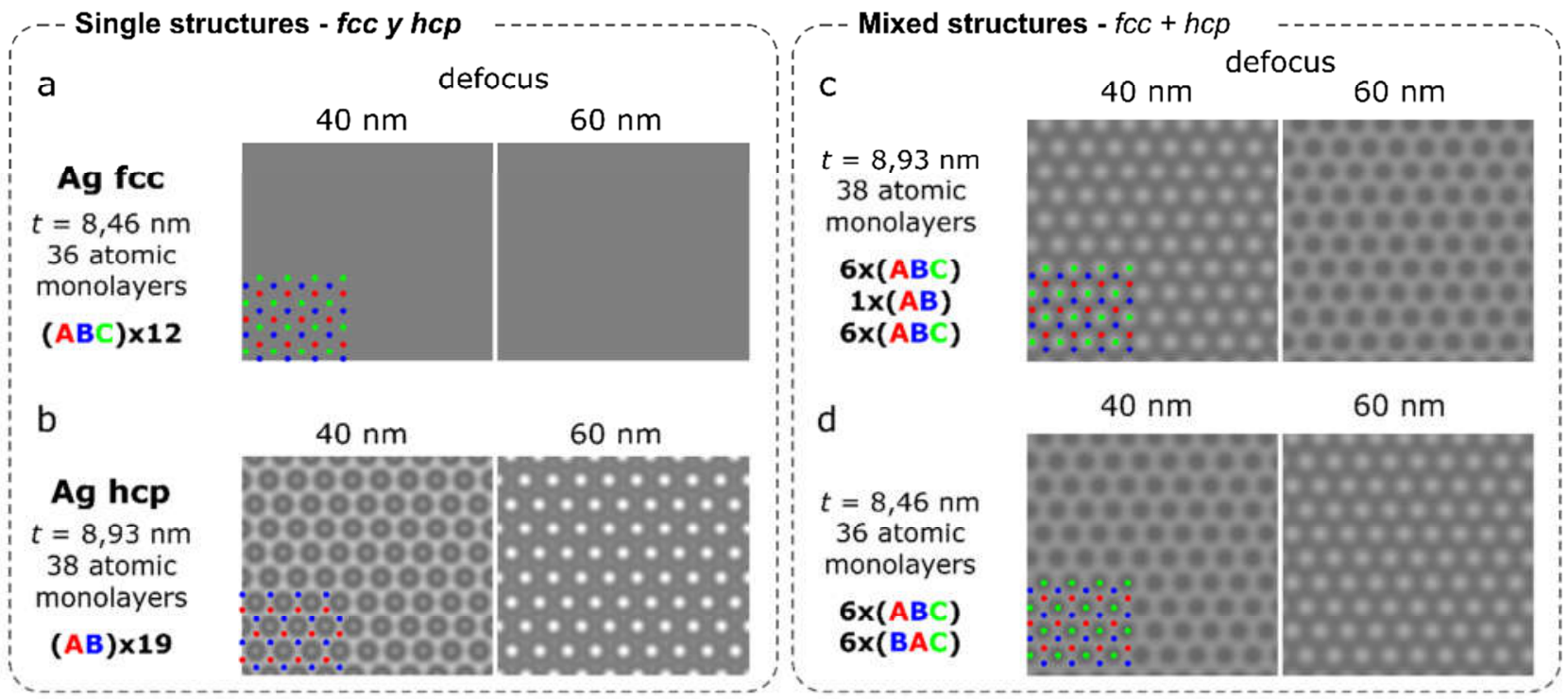

Figure 2. $\mathbf{a}$ and $\mathbf{b}$ show the simulation of fcc and hep Ag structure for different defocus values. Only b is compatible with the HRTEM images. $\mathbf{c}$ and $\mathbf{d}$ show the contrast difference of the simulation of one stacking fault or a twin. 